Check for updates

Cite this: RSC Adv., 2018, 8, 5678

Received 13th October 2017 Accepted 22nd January 2018

DOI: 10.1039/c7ra11304h

rsc.li/rsc-advances

\section{Multiwall-carbon-nanotube/cellulose composite fibers with enhanced mechanical and electrical properties by cellulose grafting}

\author{
Shaobo Zhang, (D) Feiran Zhang, Yanfei Pan, Liping Jin, Bo Liu, Yi Mao \\ and Jintian Huang (iD *
}

\begin{abstract}
Multiwall-carbon-nanotube (MWCNT)-cellulose/cellulose composite fibers with promoted mechanical and electronic activities were synthesized. Remarkably, the dispersion of MWCNTs in the composite fibers was facilitated through cellulose grafting, resulting in the tensile strength of the obtained MWCNTcellulose/cellulose composite fibers being increased to $304.6 \mathrm{MPa}$ with 10 wt\% MWCNTs involved, which was almost $106.8 \%$ higher than that of pristine MWCNT/cellulose fibers with the same amount of MWCNTs. In addition, the electrical conductivity of the MWCNT-cellulose/cellulose composite fibers was enhanced to $1.3 \times 10^{-1} \mathrm{~S} \mathrm{~cm}^{-1}$ with the dispersion of $10 \mathrm{wt} \%$ MWCNTs, which was almost 108 times higher than that of pristine MWCNT/cellulose fibers with the same amount of MWCNTs.
\end{abstract}

\section{Introduction}

Flexible electrical devices and wearable electrical appliances have been attracting significant attention due to their portability and integrability. ${ }^{1}$ As a major component of electronic devices, biocompatible and non-toxic conductive fibers have been attracting considerable attention in recent decades. For instance, they can be widely applied to wearable nano-generators, ${ }^{2}$ supercapacitors, ${ }^{3}$ batteries, ${ }^{4}$ actuators, ${ }^{5}$ and flexible solar cells. ${ }^{6}$ Cellulose, the most abundant polymer in nature, possesses a unique combination of renewable, biodegradable and biocompatible properties. Therefore, it is expected that cellulose would be considered as an inexhaustible resource to replace chemically derived compounds in some extremely desirable cases. ${ }^{7}$ However, there are some obstacles hindering the application of cellulose, such as difficult solubility owing to the large proportion of intra- and inter-molecular hydrogen bonds. Ionic liquids, a category of desirable green solvents, have been reported to be effective and promising solvents, with the merits of superior dissolving capacity, environmentally-friendly performance, recyclability, outstanding recoverability and variable structures. ${ }^{\mathbf{8}}$ It was reported that natural cellulose can dissolve in ionic liquids effectively. ${ }^{9}$ Very recently, many polymers have been applied in the preparation of hetero-structural composites in ionic liquid systems, such as films, fibers, and foams. ${ }^{10,11}$ Carbon nanotubes (CNTs) have been paid considerable attention because of their exceptional mechanical, electronic, optical, and magnetic properties. ${ }^{\mathbf{1 2 - 1 5}}$ These properties make CNTs excellent candidates to act as reinforcing agents in

College of Material Science and Art Design, Inner Mongolia Agricultural University, Hohhot, 010018, P. R. China.E-mail: jintian_h@163.com polymer composites. However, their application is hampered by the disadvantage of aggregation due to van der Waals interactions between the sidewalls of CNTs. ${ }^{16}$ Notably, the quality of the anisotropic nano-filler dispersion in the polymer matrix is directly correlated with its effectiveness for promoting mechanical and electrical functionality. Zhang synthesized regenerated-cellulose/multiwall-carbon-nanotube composite fibers in the ionic liquid 1-allyl-3-methylimidazolium chloride (AmimCl). Further exploring the mechanical and electrical activities of CNT/polymer composite fibers in ionic liquids and polymer matrix remains a challenge because of CNT aggregation. ${ }^{11}$ In previous reports, CNT/DNA fibers were prepared. The DNA facilitated the dispersion of the CNTs; the electrical conductivity of the fibers increased to $166.7 \mathrm{~S} \mathrm{~cm}^{-1}$, and the tensile strength was $101 \mathrm{MPa} .{ }^{17}$ Poulin reported dispersing CNTs in surfactant solution, recondensing the nanotubes in a flow of polymer solution to form a nanotube mesh. The

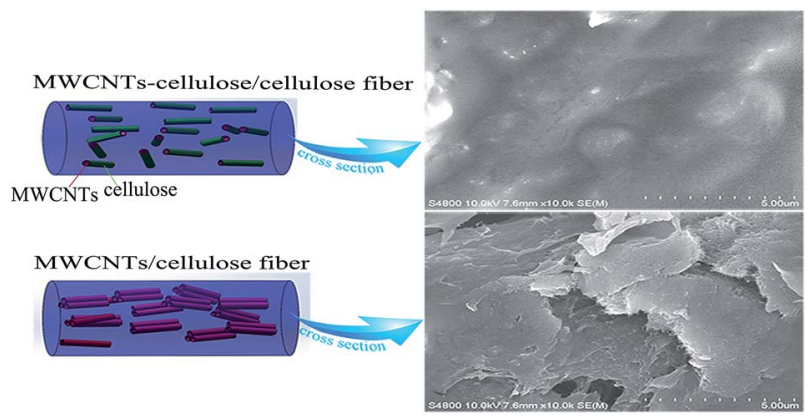

Fig. 1 Schematic diagrams of MWCNTs in a MWCNT-cellulose/ cellulose fiber and a MWCNT/cellulose fiber. 
electrical conductivity of the fibers was $10 \mathrm{~S} \mathrm{~cm}^{-1}$ at room temperature, and mean tensile strengths reached $150 \mathrm{MPa} .^{18}$

In our previous work, we developed natural polymer lignocelluloses, covalently grafting onto multiwall carbon nanotubes (MWCNTs) in the ionic liquid AmimCl, which facilitated the dispersion of MWCNTs in ionic liquids and lignocellulose films. ${ }^{19}$ Herein, MWCNT-cellulose/cellulose composite fibers with enhanced mechanical and conducting properties were prepared through cellulose being covalently grafted onto MWCNTs (Fig. 1).

\section{Experimental}

\subsection{Materials}

Salix psammophila cellulose powder was purchased from the Inner Mongolia Agricultural University, China. MWCNTs and carboxylated MWCNTs (MWCNT-COOH) were purchased from Nanjing XFNANO Co, China. AmimCl was purchased from Lanzhou Yulu Fine Chemical Co. Ltd, China. Other reagents applied in this experiment were of analytical grade.

\subsection{Preparation of MWCNT-cellulose ${ }^{19}$}

MWCNT-COOH $(0.7 \mathrm{~g})$ and $\mathrm{N}, \mathrm{N}$-dimethylformamide (DMF, $1 \mathrm{ml})$ in $\mathrm{SOCl}_{2}(30 \mathrm{ml})$ is refluxed for $24 \mathrm{~h}$; the residual $\mathrm{SOCl}_{2}$ is removed via distillation in a vacuum system. Subsequently, the obtained product is washed with acetone, filtrated and dried under vacuum. MWCNTs containing acyl chlorides (MWCNT$\mathrm{COCl})$ are obtained. Then, the above product $(5 \mathrm{mg})$ is added into AmimCl $(10 \mathrm{ml})$, sonicating for $30 \mathrm{~min}$ under vacuum. Cellulose $(0.1 \mathrm{~g})$ is mixed into AmimCl $(10 \mathrm{ml})$, stirring for $1 \mathrm{~h}$ at $50{ }^{\circ} \mathrm{C}$ under nitrogen. These two solutions are mixed together, stirring for $24 \mathrm{~h}$ at $30{ }^{\circ} \mathrm{C}$ under nitrogen. The mixture is dispersed in distilled water, sonicated, and filtrated, respectively, then dried at $50{ }^{\circ} \mathrm{C}$ under vacuum. Finally, composites containing MWCNT-cellulose are obtained.

\subsection{Preparation of MWCNT-cellulose/cellulose fibers and MWCNT/cellulose fibers}

Selected amounts of MWCNT-cellulose are added to $8 \mathrm{ml}$ of AmimCl to obtain $0 \mathrm{wt} \%, 2 \mathrm{wt} \%, 4 \mathrm{wt} \%, 6 \mathrm{wt} \%, 8 \mathrm{wt} \%, 10 \mathrm{wt} \%$, $12 \mathrm{wt} \%$ and $14 \mathrm{wt} \%$ solutions, and the mixtures are ultrasonically stirred for $30 \mathrm{~min}$ at $100{ }^{\circ} \mathrm{C}$ under vacuum. $0.6 \mathrm{~g}$ of cellulose is added into the above solutions. The mixtures are stirred for $2 \mathrm{~h}$ at $100{ }^{\circ} \mathrm{C}$ under nitrogen. MWCNT-cellulose/cellulose composite fibers are spun from the solutions using dry-jet wet-spinning. The polymer dope is transferred to a vacuum oven with degasification. Then it is heated to $100{ }^{\circ} \mathrm{C}$ for $15 \mathrm{~min}$, and extruded with nitrogen (0.5 MPa) through a single-hole spinneret $(0.8 \mathrm{~mm}$ in diameter), entering a distilled-water coagulation bath, maintained at room temperature. Subsequently, the fibers are immersed in distilled water for $24 \mathrm{~h}$ and washed with distilled water at least five times to guarantee the removal of AmimCl. The fibers are dried for $48 \mathrm{~h}$ at $80{ }^{\circ} \mathrm{C}$ under vacuum; a pre-stressing force is applied at the ends of the fibers during drying in order to improve the alignment of the fibers. For comparison, MWCNT- cellulose is replaced with MWCNTs, with the same MWCNT content, and then MWCNT/cellulose fibers are obtained.

\subsection{Characterization}

X-ray photoelectron spectroscopy (XPS) was performed using a Thermo ESCALAB 250XI multifunctional imaging electron spectrometer, at $150 \mathrm{~W}$, with an $\mathrm{Al} \mathrm{K} \alpha$ source $(h \nu=1486.6 \mathrm{eV})$. Fourier transform infrared spectra (FTIR) of the samples were collected using a Nicolet Magna-IR 750 spectrometer with a wavenumber range of $4000-400 \mathrm{~cm}^{-1}$. X-ray diffraction (XRD) was performed using an XRD diffractometer (Omicron Nanotechnology, ESCA-14, Germany), which was operated at $40 \mathrm{kV} / 30 \mathrm{~mA}$, with $\mathrm{Cu} \mathrm{K} \alpha$ radiation at a wavelength of $0.154 \mathrm{~nm}$. The Raman spectra were recorded with a Horiba LabRAM HR 800 spectroscope, using a $532 \mathrm{~nm}$ laser. Scanning electron microscopy (SEM) images of the fractured surfaces of the fibers were captured on a Hitachi S4800 microscope operating at $10 \mathrm{kV}$. The fibers were frozen in liquid nitrogen and immediately fractured. The fracture surface of the fibers was coated with a platinum layer before observation. Small-angle X-ray scattering (SAXS) measurements were obtained using a NanoSTAR U small-angle X-ray scattering system (Bruker, Germany), using a microfocus source with $\mathrm{Cu}-\mathrm{K} \alpha$ radiation $(45 \mathrm{kV}, 0.65 \mathrm{~mA})$. The mechanical properties of the MWCNT/cellulose and MWCNTcellulose/cellulose composite fibers were measured using a universal testing machine (5940 Instron, USA) at a crosshead speed of $10 \mathrm{~mm} \mathrm{~min}^{-1}$. A gauge length of $20 \mathrm{~mm}$ was used. Mechanical tests were performed at room temperature. To ensure data accuracy and repeatability, at least five measurements were carried out for each composite fiber. The electrical conductivity of the fibers was measured at room temperature using a Solartron 7081 precision voltmeter with a four-probe method.

\section{Results and discussion}

\subsection{Structure of MWCNT-cellulose}

As shown in Fig. 2, the XPS survey spectrum demonstrated the following element content values: MWCNT-COCl had 98.45 at\% carbon, 1.03 at\% oxygen and 0.52 at\% chlorine content; and MWCNT-cellulose had 90.13 at\% carbon and 9.87 at\% oxygen content. For further confirmation of the composite structure, the characteristic C 1s peaks of MWCNT-cellulose were deconvoluted via Gaussian fitting (Fig. 2b). The $\mathrm{C} 1 \mathrm{~s}$ peak at $289.2 \mathrm{eV}$ was ascribed to the ester carbon $(\mathrm{O}=\mathrm{C}-\mathrm{O}){ }^{20}$ The $\mathrm{C}$ 1s peaks at $287.5 \mathrm{eV}$ and $286.6 \mathrm{eV}$ arose from the bridging carbon $(\mathrm{O}-\mathrm{C}-\mathrm{O})$ in the glucopyranose rings of cellulose and $\mathrm{C}-\mathrm{O}$ in the ester groups and cellulose. ${ }^{21,22}$ The $\mathrm{C} 1 \mathrm{~s}$ peaks at $285.6 \mathrm{eV}$ and $285.4 \mathrm{eV}$ represented the $\mathrm{sp}^{2}$ and $\mathrm{sp}^{3}$ hybridization of the $\mathrm{C}$ atom. ${ }^{23}$ In addition, the chlorine element of MWCNT-COCl and MWCNT-cellulose samples was investigated via XPS; the Cl 2p spectrum is exhibited in Fig. 1c. The $\mathrm{Cl} 2$ p peaks at $201.8 \mathrm{eV}$ and $200.2 \mathrm{eV}$ arose from the acyl chloride group $(\mathrm{O}=\mathrm{C}-\mathrm{Cl})$ in MWCNT-COCl. However, there was almost no chlorine in MWCNT-cellulose, indicating that the acyl chloride group was involved in the reaction between MWCNT-COCl and cellulose. 


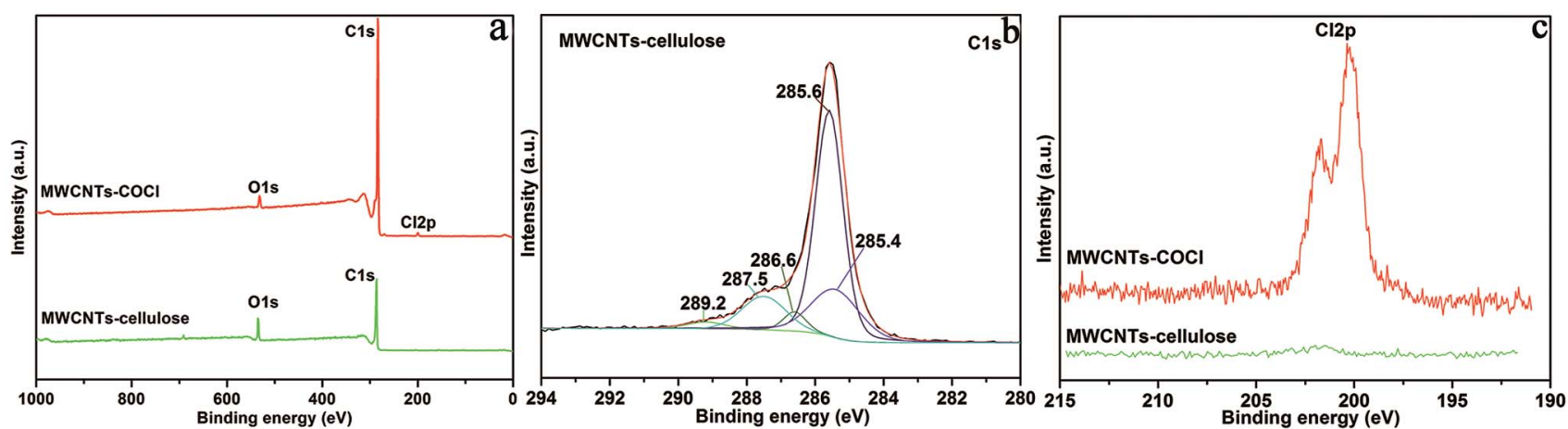

Fig. 2 (a) XPS survey scan of MWCNT-COCl and MWCNT-cellulose. (b) XPS spectrum of the C 1s core level of MWCNT-cellulose. The spectrum curves were deconvoluted via Gaussian fitting. (c) Cl 2p narrow XPS scan of MWCNT-COCl and MWCNT-cellulose.

These XPS results indicated that cellulose was grafted onto the MWCNTs via an esterification reaction.

Fig. 3 exhibits FTIR spectra of MWCNT-COCl, MWCNT$\mathrm{COOH}$, MWCNT-cellulose and cellulose. The spectrum of cellulose shows a peak at $900 \mathrm{~cm}^{-1}$, which is indicative of betaD-glucopyranosyl. Strong peaks at $1000-1200 \mathrm{~cm}^{-1}$ corresponded to the $\mathrm{C}-\mathrm{O}$ stretching of the superposition of $\mathrm{C}-\mathrm{O}-\mathrm{C}$ groups, which was intra and between the anhydroglucose ring and the $\mathrm{C}-\mathrm{O}$ of the secondary hydroxyl and primary oxhydryl. The pointed transmittances at $1116 \mathrm{~cm}^{-1}$ and $1037 \mathrm{~cm}^{-1}$ were ascribed to the $\mathrm{C}-\mathrm{O}$ stretching of the secondary hydroxyl and primary oxhydryl, respectively. The peak at $2902 \mathrm{~cm}^{-1}$ was ascribed to $\mathrm{C}-\mathrm{H}$ bond stretching. The broad peak at $3422 \mathrm{~cm}^{-1}$ was related to hydroxyl groups and hydrogen bonds. The spectrum of MWCNT-COOH (Fig. 3b) demonstrated that the peak at $1089 \mathrm{~cm}^{-1}$ could be ascribed to carboxyl C-O bond stretching. The transmittance at $1725 \mathrm{~cm}^{-1}$ was ascribed to carboxyl $\mathrm{C}=\mathrm{O}$ bond stretching. The transmittance at $3438 \mathrm{~cm}^{-1}$ was related to $\mathrm{O}-\mathrm{H}$ bond stretching. After acylation, as shown in Fig. $3 \mathrm{a}$, in the FTIR spectrum of MWCNT-COCl the transmittance at $3438 \mathrm{~cm}^{-1}$ seen in MWCNT-COOH was weakened, and new

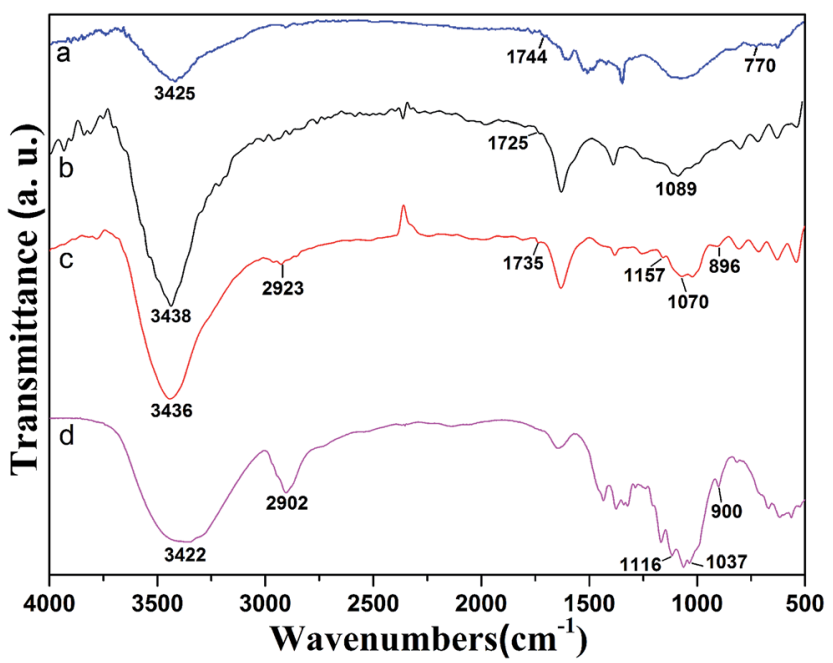

Fig. 3 FTIR spectra of MWCNT-COCl (a), MWCNT-COOH (b), MWCNT-cellulose (c), and cellulose (d). transmittances at $1744 \mathrm{~cm}^{-1}$ and $770 \mathrm{~cm}^{-1}$ were ascribed to $\mathrm{C}=\mathrm{O}$ and $\mathrm{C}-\mathrm{Cl}$ in the acyl chloride group. After modification with cellulose, in the FTIR spectrum of MWCNT-cellulose (Fig. 3c), the characteristic bands from cellulose appeared at $2923 \mathrm{~cm}^{-1}$ and $896 \mathrm{~cm}^{-1}$, which were respectively ascribed to $\mathrm{C}-\mathrm{H}$ bond stretching and beta-D-glucopyranosyl. Moreover, the transmittances at $1116 \mathrm{~cm}^{-1}$ and $1037 \mathrm{~cm}^{-1}$ from cellulose were absent after modification. This could be triggered by the secondary hydroxyl and primary oxhydryl in the reaction system. Additionally, in the FTIR spectrum of MWCNT-cellulose (Fig. 3c), the transmittance at $770 \mathrm{~cm}^{-1}$ from MWCNT-COCl was absent, caused by the $\mathrm{C}-\mathrm{Cl}$ of the acyl chloride group being involved in the reaction. New transmittances from MWCNTcellulose at $1735 \mathrm{~cm}^{-1}, 1070 \mathrm{~cm}^{-1}$ and $1157 \mathrm{~cm}^{-1}$ were ascribed to $\mathrm{C}=\mathrm{O}$ and $\mathrm{C}-\mathrm{O}-\mathrm{C}$ bond stretching of the ester. The transmittance results indicate the formation of covalent bonds between cellulose and MWCNTs.

XRD studies of the MWCNTs, MWCNT-COOH, MWCNTCOCl, cellulose, MWCNT-cellulose, cellulose fibers, MWCNT/ cellulose fibers and MWCNT-cellulose/cellulose fibers were done to investigate the crystalline behaviors of the samples (Fig. 4). The MWCNT X-ray diffraction pattern shows a $2 \theta$ diffraction peak angle of about $26^{\circ}$, which is characteristic of the (200) plane reflection of a graphite structure. This sharp peak indicates the neat arrangement of $\mathrm{C}$ atoms in the MWCNTs. After carboxylation and acylation (Fig. $4 \mathrm{~b}$ and c), the full width at half maximum (FWHM) of the sharp characteristic diffraction peak from the MWCNTs was bigger. This indicates that the crystallite sizes were smaller. This could be triggered by some neat structure in the MWCNTs being broken in the processes of carboxylation and acylation. In the spectrum of cellulose (Fig. 4d), the peaks at $16.4^{\circ}$ from (101) plane reflection and $22.5^{\circ}$ from (002) plane reflection were caused by the transverse arrangement of the crystallites in cellulose $\mathrm{I}^{24}$ Fig. 4e-h show X-ray diffraction patterns of MWCNT-cellulose, cellulose fibers, MWCNT/cellulose fibers and MWCNTcellulose/cellulose fibers. All samples showed the typical cellulose II crystalline form. ${ }^{25}$ This indicated that the cellulose could dissolve completely in AmimCl. In the spectrum of MWCNTcellulose (Fig. $4 \mathrm{~d}$ ), the peaks at $21.4^{\circ}$ and $26^{\circ}$ were from the cellulose II crystalline form and graphite structure of MWCNTs, 


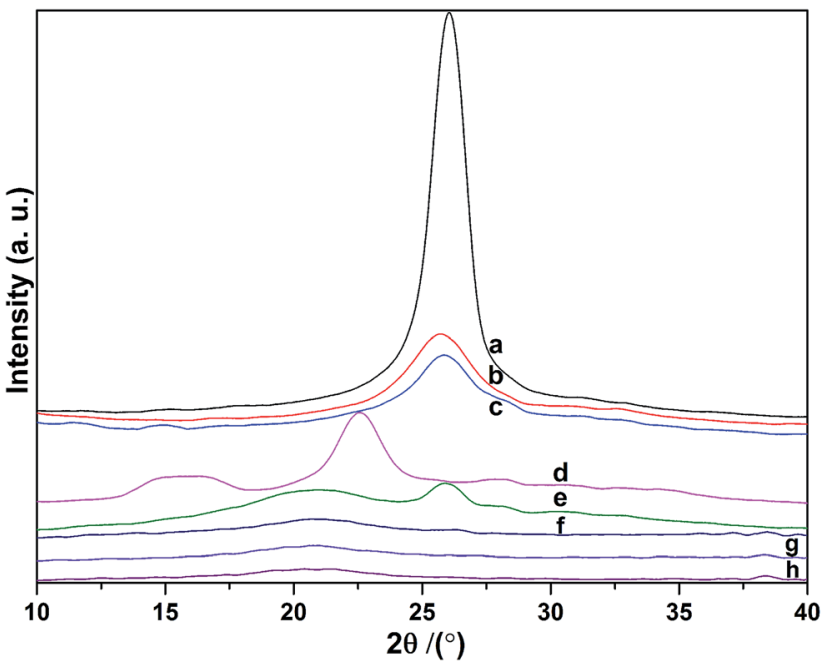

Fig. 4 XRD analysis of MWCNTs (a), MWCNT-COOH (b), MWCNT$\mathrm{COCl}$ (c), cellulose (d), MWCNT-cellulose (e), cellulose fibers (f), MWCNT/cellulose fibers (10 wt\% MWCNTs) (g), and MWCNT-cellulose/cellulose fibers (10 wt\% MWCNTs) (h).

respectively. In addition, as shown in the spectra of the cellulose fibers, MWCNT/cellulose fibers and MWCNT-cellulose/cellulose fibers, the crystallinity of the cellulose fibers was higher than that of the MWCNT/cellulose fibers, and the crystallinity of the MWCNT/cellulose fibers was higher than that of the MWCNTcellulose/cellulose fibers. This was caused by MWCNTs hindering the formation of crystalline regions among the cellulose chains, leading to a decrease in the degree of crystallinity. ${ }^{26}$ The MWCNTs in the MWCNT-cellulose/cellulose fibers were more uniform than in the MWCNT/cellulose fibers.

Raman spectra of MWCNTs over the range of 1200$1800 \mathrm{~cm}^{-1}$ were dominated by two peaks: the D-band (at $1337 \mathrm{~cm}^{-1}$, attributed to disorder induced by defects and curvature in the nanotube lattice) and the G-band (at $1577 \mathrm{~cm}^{-1}$, due to the in-plane vibration of $\mathrm{C}-\mathrm{C}$ bonds). ${ }^{27}$ The integral area ratio of the D-band to the G-band $\left(I_{\mathrm{D}} / I_{\mathrm{G}}\right)$ can be used to evaluate the extent of any carbon-containing defects. ${ }^{28}$ Raman spectra of the samples are shown in Fig. 5. Compared with $I_{\mathrm{D}} / I_{\mathrm{G}}$ of MWCNTs (1.1), $I_{\mathrm{D}} / I_{\mathrm{G}}$ of MWCNT-COOH was higher (1.4). This indicates that the MWCNTs were destroyed during the carboxylation procedure. However, there was no obvious change in the values of MWCNT-COCl and MWCNT-cellulose, compared with MWCNT-COOH. This demonstrated that during the acylation procedure the MWCNTs were not obviously destroyed. The intensities of $I_{\mathrm{D}}$ and $I_{\mathrm{G}}$ from MWCNT-cellulose were lower; this could be caused by the MWCNTs being pressured by "coating layer" cellulose, which limited particle vibration. ${ }^{29}$ Due to the MWCNT content in MWCNT/cellulose fibers and MWCNT-cellulose/cellulose fibers being low, the Dband and G-band were weaker. However, the D-band and Gband from the MWCNT-cellulose/cellulose fibers were stronger than from the MWCNT/cellulose fibers; this could be caused by the uniform MWCNTs in the MWCNT-cellulose/ cellulose fibers.

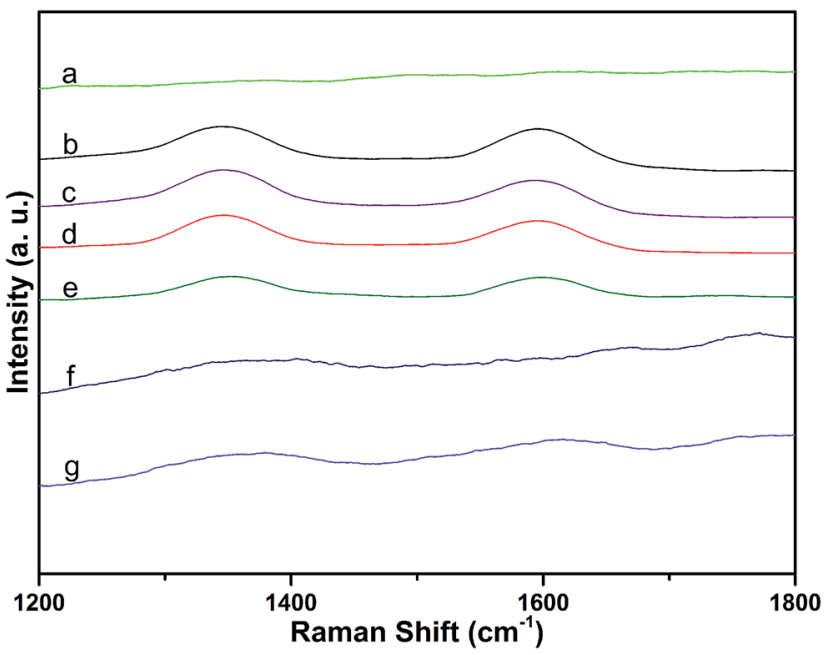

Fig. 5 Raman spectra of cellulose (a), MWCNTs (b), MWCNT-COOH (c), MWCNT-COCl (d), MWCNT-cellulose (e), MWCNT/cellulose fibers (10 wt\% MWCNTs) (f), and MWCNT-cellulose/cellulose fibers (10 wt\% MWCNTs) (g).

\subsection{Morphology and structure of MWCNT/cellulose fibers and MWCNT-cellulose/cellulose fibers}

Fig. 6 shows photographs and SEM images of the cellulose fibers, MWCNT/cellulose fibers with $10 \mathrm{wt} \%$ MWCNTs, and MWCNTcellulose/cellulose composite fibers (containing $10 \mathrm{wt} \%$ MWCNTs). The cellulose fibers were nearly transparent. The cross sectional surface of the pure cellulose fibers was flat and featureless in general, illustrating that the resulting cellulose fibers were brittle and the cracks propagate in a planar fashion. However, for the MWCNT/cellulose fibers, the cross sectional surface was extremely rough, which was rather clearly distinctive, compared to the flat fracture surface of the pure cellulose fibers. This indicated that the MWCNTs played a significant role in improving the mechanical properties of the cellulose fibers. Some irregular agglomerated MWCNTs in the cellulose matrix were observed subsequently. The mechanical and electrical properties of the composite fibers would be slightly hampered owing to this aggregation. Remarkably, there were almost no agglomerated particles in the cross sectional surface of the MWCNT-cellulose/ cellulose fibers. A homogenous dispersion of MWCNT particles was observed predominantly, compared with the agglomerated particles of the MWCNT/cellulose fibers. The images in Fig. 6d and $\mathrm{g}$ show the MWCNTs and MWCNT-cellulose in AmimCl after 30 days of storage, respectively. In Fig. $6 \mathrm{~d}$, a large quantity of black MWCNT precipitate is observed in the MWCNT dispersion, whereas the MWCNT-cellulose appears uniform without any MWCNT precipitate (Fig. 6g). These results indicate that the stability of a MWCNT-cellulose dispersion was superior to MWCNTs in AmimCl. These results suggest that cellulose was covalently grafted onto the surface of the MWCNTs, facilitating the dispersion of MWCNTs in the cellulose matrix, and the miscibility between MWCNTs and the cellulose matrix was dramatically promoted via MWCNTs wrapped with cellulose. The quality of the anisotropic nano-filler dispersion in the polymer 


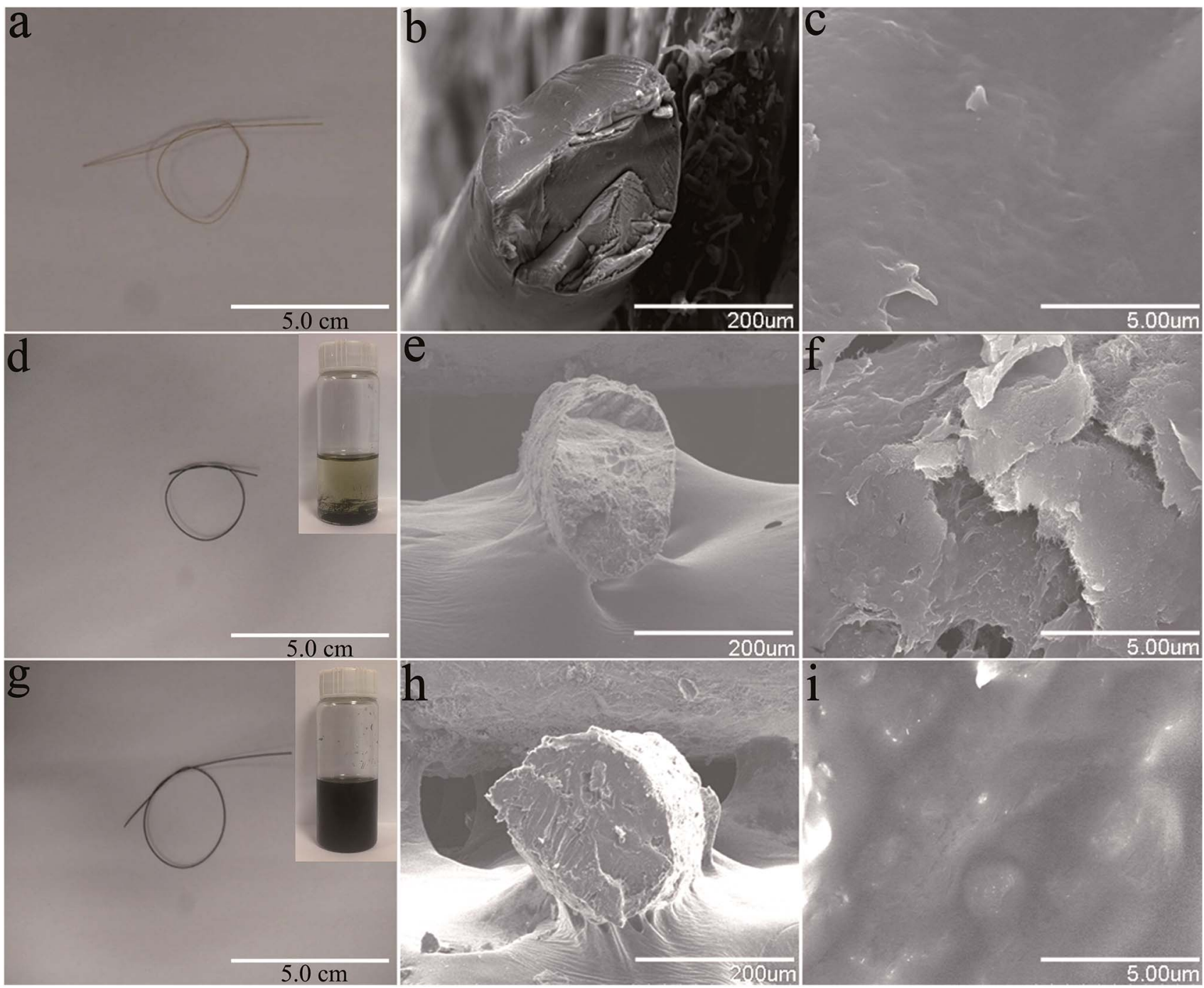

Fig. 6 (a, $d$ and g) Photographs of a cellulose fiber, MWCNT/cellulose fiber (10 wt\% MWCNTs), and MWCNT-cellulose/cellulose composite fiber (10 wt\% MWCNTs). (b, c) SEM images of a cross-sectional fracture of the cellulose fiber. (e, f) SEM images of a cross-sectional fracture of the MWCNT/cellulose fiber (10 wt\% MWCNTs). (h, i) SEM images of a cross-sectional fracture of the MWCNT-cellulose/cellulose composite fiber (10 wt\% MWCNTs). The inset images in (d) and (g) show MWCNTs and MWCNT-cellulose in AmimCl after 30 days of storage. The concentration of MWCNTs is $0.125 \mathrm{mg} \mathrm{ml}^{-1}$.

matrix was rather modified in its effectiveness in enhancing mechanical and electrical activities.

SAXS patterns of MWCNT-cellulose/cellulose fibers, MWCNT/cellulose fibers and cellulose fibers are exhibited in Fig. 7. As the MWCNTs join, the intensity of the SAXS diffraction of the fibers increases. However, the SAXS diffraction intensity of MWCNT-cellulose/cellulose fibers is higher than MWCNT/ cellulose fibers; this could be caused by irregular agglomerated particles in the MWCNT/cellulose fibers reducing the intensity of the SAXS diffraction, compared with the MWCNTcellulose/cellulose fibers.

\subsection{Mechanical properties of MWCNT/cellulose fibers and MWCNT-cellulose/cellulose fibers}

Furthermore, typical tensile strengths of MWCNT/cellulose fibers, MWCNT-cellulose/cellulose fibers and cellulose fibers were collected respectively, as shown as Fig. 8. The tensile strength of MWCNT/cellulose fibers increased to approximately 203.4 MPa with $6 \mathrm{wt} \%$ MWCNTs. However, with MWCNT loading further increasing, the tensile strength decreased inversely, which might be caused by the aggregation of MWCNTs in increasing amounts. For the MWCNT-cellulose/ cellulose composite fibers, with an increase in MWCNTcellulose involved, the tensile strength dramatically improved to $304.6 \mathrm{MPa}$ with $10 \mathrm{wt} \%$ MWCNTs, which was almost $106.8 \%$ higher than that of MWCNT/cellulose fibers with the same amount of MWCNTs. Meanwhile, this was $117.1 \%$ higher than that of cellulose fibers. Moreover, the tensile strength of the MWCNT-cellulose/cellulose fibers was much higher than that of MWCNT/cellulose fibers with the same amount of MWCNTs. The possible reason was that the cellulose covalently grafted along the surface of the MWCNTs facilitated the dispersion of 


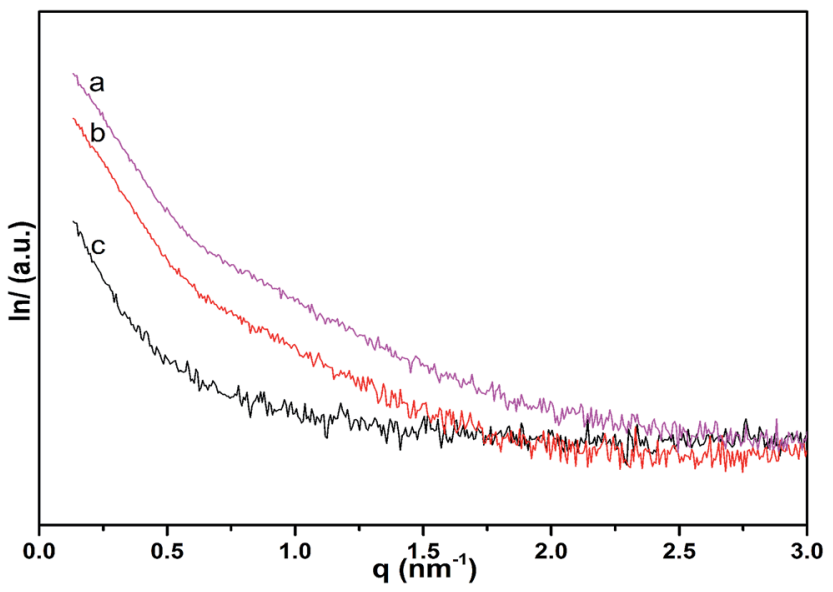

Fig. 7 SAXS patterns of MWCNT-cellulose/cellulose fibers (10 wt\% MWCNTs) (a), MWCNT/cellulose fibers (10 wt\% MWCNTs) (b), and cellulose fibers (c).

MWCNTs in AmimCl and the cellulose matrix, thus leading to the highly promoted tensile strength performance.

\subsection{Electrical properties of MWCNT/cellulose fibers and MWCNT-cellulose/cellulose fibers}

The electrical conductivity values of the MWCNT/cellulose fibers, MWCNT-cellulose/cellulose fibers and cellulose fibers are shown in Table 1. In terms of MWCNT/cellulose fibers containing 6 wt $\%$ MWCNTs, it was demonstrated that the electrical conductivity was up to $8.0 \times 10^{-3} \mathrm{~S} \mathrm{~cm}^{-1}$. However, the electrical conductivity values obtained for MWCNT/ cellulose fibers containing more than 6 wt $\%$ MWCNTs declined, indicating that involving a large amount of MWCNTs could not dramatically increase electrical conductivity, possibly due to aggregation and poor dispersion. ${ }^{11}$ Similar phenomena were observed in a PC/MWCNT fiber composite, as reported by Potschke. ${ }^{30}$ Comparably, the electrical conductivity of MWCNTcellulose/cellulose fibers improved with the loading of MWCNT-

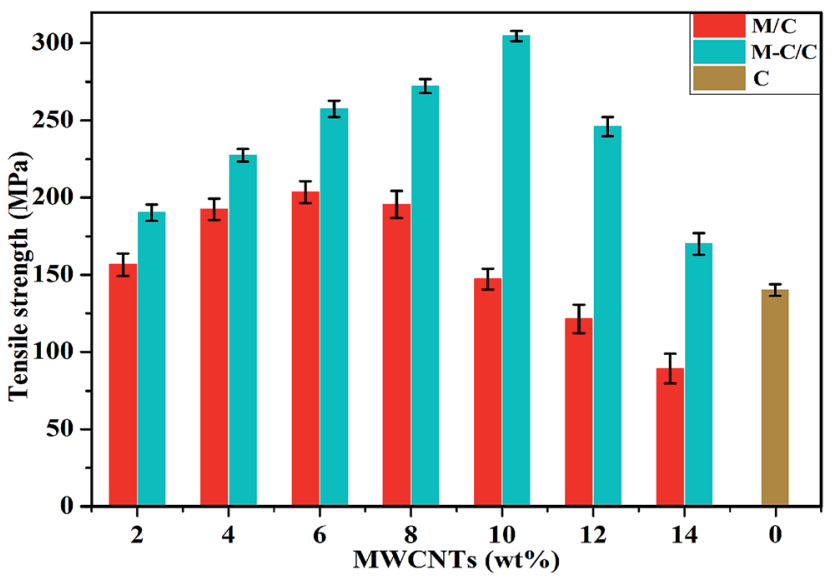

Fig. 8 Tensile strengths of MWCNT/cellulose composite fibers (M/C), MWCNT-cellulose/cellulose composite fibers (M-C/C), and cellulose fibers (C).
Table 1 Electrical conductivity values for MWCNT/cellulose fibers and MWCNT-cellulose/cellulose fibers

\begin{tabular}{lll}
\hline & $\begin{array}{l}\text { MWCNT-cellulose/cellulose } \\
\text { fibers }\left(\mathrm{S} \mathrm{cm}^{-1}\right)\end{array}$ & $\begin{array}{l}\text { MWCNT/cellulose } \\
\text { fibers }\left(\mathrm{S} \mathrm{cm}^{-1}\right)\end{array}$ \\
\hline 0 & 0 & 0 \\
2 & $4.2 \times 10^{-4}$ & $1.3 \times 10^{-5}$ \\
4 & $2.1 \times 10^{-2}$ & $2.8 \times 10^{-3}$ \\
6 & $6.8 \times 10^{-2}$ & $8.0 \times 10^{-3}$ \\
8 & $8.1 \times 10^{-2}$ & $4.1 \times 10^{-3}$ \\
10 & $1.3 \times 10^{-1}$ & $1.2 \times 10^{-3}$ \\
12 & $0.9 \times 10^{-2}$ & $9.3 \times 10^{-4}$ \\
14 & $7.2 \times 10^{-3}$ & $6.5 \times 10^{-5}$
\end{tabular}

cellulose, up to approximately $1.3 \times 10^{-1} \mathrm{~S} \mathrm{~cm}^{-1}$ with $10 \mathrm{wt} \%$ MWCNTs, which was almost 108 times higher than that of MWCNT/cellulose fibers with the same amount of MWCNTs. Furthermore, the electrical conductivity of the MWCNTcellulose/cellulose fibers was higher than that of the MWCNT/ cellulose fibers for each amount of MWCNTs. They were dramatically superior to MWCNT/cellulose fibers because of the improved stability from the dispersion of MWCNT-cellulose in AmimCl and the cellulose matrix.

\section{Conclusions}

Well-designed MWCNT-cellulose/cellulose fibers with enhanced mechanical and electrical properties were fabricated in AmimCl. XPS spectra of MWCNT-COCl and MWCNTcellulose were investigated. FTIR data from MWCNT-COCl, MWCNT-COOH, MWCNT-cellulose and cellulose were collected. XRD data from MWCNTs, MWCNT-COOH, MWCNTCOCl, cellulose, MWCNT-cellulose, cellulose fibers, MWCNT/ cellulose fibers and MWCNT-cellulose/cellulose fibers were investigated. Raman spectra of cellulose, MWCNTs, MWCNT$\mathrm{COOH}$, MWCNT-COCl, MWCNT-cellulose, MWCNT/cellulose fibers and MWCNT-cellulose/cellulose fibers were recorded. SAXS patterns of MWCNT-cellulose/cellulose fibers, MWCNT/ cellulose fibers and cellulose fibers were investigated. The cross sectional structures of the above composite fibers were investigated using SEM. Cellulose grafting onto the surfaces of the MWCNTs facilitated the dispersion of MWCNTs in the cellulose matrix. The tensile strengths of the obtained MWCNTcellulose/cellulose composite fibers and MWCNT/cellulose fibers were measured systemically. The tensile strength of the MWCNT/cellulose fiber composite was up to approximately 203.4 MPa with 6 wt\% MWCNTs. Comparably, the tensile strength of the MWCNT-cellulose/cellulose fiber composite increased to $304.6 \mathrm{MPa}$ with $10 \mathrm{wt} \%$ MWCNTs, which was almost 106.8\% higher than that of MWCNT/cellulose fibers with the same amount of MWCNTs. In addition, the electrical performances of MWCNT/cellulose fibers and MWCNTcellulose/cellulose composite fibers were studied. The highest electrical conductivity of the MWCNT/cellulose fibers was up to $8.0 \times 10^{-3} \mathrm{~S} \mathrm{~cm}^{-1}$ with $6 \mathrm{wt} \%$ MWCNTs, while the highest electrical conductivity of the MWCNT-cellulose/cellulose composite fibers was promoted to $1.3 \times 10^{-1} \mathrm{~S} \mathrm{~cm}^{-1}$ with 
$10 \mathrm{wt} \%$ MWCNTs, which was almost 108 times higher than that of MWCNT/cellulose fibers with the same amount of MWCNTs. Therefore, the well-designed MWCNT-cellulose/cellulose composite fibers could open a new path for applications of wearable electrical devices.

\section{Conflicts of interest}

There are no conflicts to declare.

\section{Acknowledgements}

This work was supported by the National Key Technology Research and Development Program of the Ministry of Science and Technology of China under Grant no. 2015BAC06B02-3, the Science Research Innovation Projects of the Inner Mongolia Autonomous Region for Graduates under Grant no. B20161012909 and the Natural Science Foundation of the Inner Mongolia Autonomous Region under Grant no. 2016MS0353.

\section{References}

1 J. Ren, W. Bai, G. Guan, Y. Zhang and H. Peng, Adv. Mater., 2013, 25, 5965-5970.

2 M. Lee, C. Y. Chen, S. Wang, S. N. Cha, Y. J. Park, J. M. Kim, L. J. Chou and Z. L. Wang, Adv. Mater., 2012, 24, 1759-1765.

3 J. A. Lee, K. S. Min, H. K. Shi, H. U. Cho, G. M. Spinks, G. G. Wallace, M. D. Lima, X. Lepro, M. E. Kozlov, R. H. Baughman and J. K. Seon, Nat. Commun., 2013, 4, 1970-1978.

4 J. Ren, L. Li, C. Chen, X. Chen, Z. Cai, L. Qiu, Y. Wang, X. Zhu and H. Peng, Adv. Mater., 2013, 25, 1155-1162.

5 H. Cheng, J. Liu, Y. Zhao, C. Hu, Z. Zhang, N. Chen, L. Jiang and L. Qu, Angew. Chem., Int. Ed., 2013, 52, 10482-10486.

6 T. Chen, S. Wang, Z. Yang, Q. Feng, X. Sun, L. Li, Z. S. Wang and H. Peng, Angew. Chem., Int. Ed., 2011, 50, 1815-1824.

7 D. Klemm, B. Heublein, H.-P. Fink and A. Bohn, Angew. Chem., Int. Ed., 2005, 117, 3422-3458.

8 S. S. Y. Tan and D. R. MacFarlane, Top. Curr. Chem., 2009, 290, 311-339.

9 I. Kilpeläinen, H. Xie, A. King, M. Granstrom, S. Heikkinen and D. S. Argyropoulos, J. Agric. Food Chem., 2007, 55, 9142-9148.
10 Z. Luo, A. Wang, C. Wang, W. Qin, N. Zhao, H. Song and J. Gao, J. Mater. Chem. A, 2014, 2, 7327-7336.

11 H. Zhang, Z. Wang, Z. Zhang, J. Wu, J. Zhang and J. He, Adv. Mater., 2007, 19, 698-704.

12 A. Bianco, K. Kostarelos, C. D. Partidos and M. Prato, Chem. Commun., 2005, 571-577.

13 H. Dai, Acc. Chem. Res., 2002, 35, 1035-1044.

14 S. Iijima, Nature, 1991, 354, 56-58.

15 A. Jorio, G. Dresselhaus and M. S. Dresselhaus, J. Mol. Evol., 2008, 74, 52-60.

16 R. Liu, X. Zeng, J. Liu, Y. Zheng, J. Luo and X. Liu, J. Mater. Chem. A, 2014, 2, 14481-14492.

17 J. N. Barisci, M. Tahhan, G. G. Wallace, S. Badaire, T. Vaugien, M. Maugey and P. Poulin, Adv. Funct. Mater., 2004, 14, 133-138.

18 B. Vigolo, A. Penicaud, C. Coulon, C. sauder, R. Pailler, C. Journet, P. Bernier and P. Poulin, Science, 2000, 290, 1331-1334.

19 J. T. Huang, S. B. Zhang, F. R. Zhang, Z. Q. Guo, L. P. Jin, Y. F. Pan, Y. Wang and T. C. Guo, Carbohydr. Polym., 2017, 160, 115-122.

20 G. Beamson and D. Briggs, High resolution XPS of organic polymers: the Scienta ESCA300 database, Wiley, 1992.

21 N. M. D. Brown, J. A. Hewitt and B. J. Meenan, Surf. Interface Anal., 1992, 18, 187-198.

22 G. H. Gao, Y. H. Lei, L. H. Dong, W. C. Liu, X. F. Wang, X. T. Chang, T. Liu, Y. S. Yin and M. A. Pulickel, Mater. Express, 2012, 2, 1-9.

23 X. D. He, F. H. Zhang, R. G. Wang and W. B. Liu, Carbon, 2007, 45, 2559-2563.

24 A. E. Zavadskii, Fibre Chem., 2004, 36, 425-430.

25 H. Qi, C. Chang and L. Zhang, Green Chem., 2009, 11, 177184.

26 H. Qi, J. Liu, S. Gao and E. Mäder, J. Mater. Chem. A, 2013, 1, 2161-2168.

27 D. Baskaran, J. W. Mays and M. S. Bratcher, Chem. Mater., 2005, 17, 3389-3397.

28 L. Li, L. Meng, X. Zhang, C. Fu and Q. Lu, J. Mater. Chem., 2009, 19, 3612-3617.

29 C. Stephan, T. P. Nguyen, M. Lamy de la Chapelle, S. Lefrant, C. Journet and P. Bernier, Synth. Met., 2000, 108, 139-149.

30 P. Potschke, H. Brunig, A. Janke, D. Fischer and D. Jehnichen, Polymer, 2005, 46, 10355-10363. 